\title{
Kulturlandschaftswandel in der heutigen Schweiz als Verlust von Heimat*
}

\begin{abstract}
These
Der Schweiz war es im 19. und 20. Jahrhundert gelungen, die europäische Industrialisierung auf eine eigenständige Weise zu gestalten, was sich u.a. daran zeigte, daß die traditionellen Kulturlandschaften verändert, aber nicht zerstört wurden. Mit der ab 1955 einsetzenden Tertiarisierung steht sie aber vor einer neuen, noch größeren Herausforderung: Das bestehende kulturelle Selbstbewußtsein und das bisher bewährte politische Instrumentarium reicht zu Beginn der 90er Jahre nicht mehr aus, um die von den europäischen Zentren mitgesteuerte Tertiarisierung in der Schweiz auf eine spezifisch helvetische Weise zu prägen und damit «Heimat» aktiv zu gestalten. Daher ist eine Selbstbesinnung erforderlich, die im Rückgriff und in Neuinterpretation der eigenen Vergangenheit die Vision einer zukünftigen Schweiz ("Leitbild Schweiz») entwirft, die sich weder haltlos der Tertiarisierung unterwirft noch isolationistisch davon abzuschotten versucht.
\end{abstract}

\section{Die schweizerische Form der Industrialisierung}

Die Schweiz trat mit einer «vormodernen» Staatsstruktur in die Phase der Industrialisierung ein, nämlich einem dem Nationalstaatsgedanken widersprechenden Konglomerat verschiedener Sprach-, Kultur-, Volks- und Religionsgruppen. Mit der Umwandlung vom Staatenbund zum Bundesstaat im Jahre 1848 wurde die für das Industriezeitalter erforderliche neue Staatsform aufgebaut, ohne gleichzeitig mit zentralen Werten der Vergangenheit (Beibehaltung der gewachsenen kleinräumigen Unterschiede, starke föderalistisch-dezentrale Prägung)

\footnotetext{
*Angesichts des essayistischen Charakters dieses Beitrages und der gebotenen Kürze wurde darauf verzichtet, die Ausführungen mit empirischen Daten und Literaturverweisen zu versehen. Die vier nationalen Forschungsprogramme "MAB", "Regio", "Boden" und "Stadt" enthalten sehr viele empirische Analysen zur Stützung dieser Aussagen, und es wäre sinnvoll, alle vier Programme unter der skizzierten Leitfrage zusammenfassend auszuwerten, denn sie decken relevante Bereiche der schweizerischen Realität in bezug auf die Tertiarisierung ab.
}

zu brechen. Während die Industrialisierung in den großen Nationalstaaten Europas zu markanten räumlichen Disparitäten durch Entwertung der ländlichen Räume (Reagrarisierung durch Zusammenbruch aller nichtlandwirtschaftlicher Wirtschaftszweige) und Aufwertung der Städte (als Industriezentren bzw. -reviere) führte, zeichnet sich die schweizerische Industrialisierung durch eine relativ dezentrale Gestaltung aus. Dies liegt zwar auch an naturräumlichen Voraussetzungen (Fehlen von Kohle-/Erzlagern, damit schlechte Voraussetzungen für die räumlich hochkonzentrierte Schwerindustrie; dagegen Existenz eines dezentralen Gewässernetzes, das eine dezentrale Wasserkraftnutzung nahelegt), aber entscheidender dafür war die föderalistische Staatsstruktur sowie das außenpolitische Selbstverständnis (kein staatlich forcierter Aufbau einer Schwerindustrie aus machtpolitischen Gründen). Charakteristisch für die Phase der Industriegesellschaft ist es, daß eigentliche Industriegebiete fehlen und da $ß$ sich kein Wirtschaftszentrum herausbildet, das die gesamte Schweiz dominiert. Diese Entwicklung findet auf der gesellschaftlichen Ebene ihre Entsprechung darin, daß der Gegensatz zwischen «Kapital» und «Arbeit» schwächer ist als in den anderen Industriestaaten; das 1937 abgeschlossene «Friedensabkommen", das bis heute gilt, stellt ein singuläres Faktum in ganz Europa dar. Auch wenn die für das Industriezeitalter charakteristischen räumlichen, sozialen, politischen Widersprüche in der Schweiz weniger stark ausgeprägt sind als sonst in Europa und dadurch vorindustrielle Lebens-, Wirtschafts- und Kulturlandschaftsformen besser als anderswo im neuen Rahmen weiterleben und mit ihm verbunden werden können, so kann sich die Schweiz doch nicht den fundamentalen Veränderungen entziehen: Die Disparitäten zwischen Stadt und Land brechen auch hier auf und führen zur Entwertung der peripheren und Gebirgs-Regionen bzw. zur Aufwertung von zentralen Teilen des Mittellandes; allerdings sind diese Disparitäten nicht so stark ausgeprägt, als daß die Schweiz dadurch ihre Identität und ihren eigenständigen Charakter verliert.

Werner Bätzing, Dr., Geogr. Institut der Universität Bern, Hallerstraße 12, 3012 Bern. 


\section{Tertiarisierung}

Unter Tertiarisierung versteht man die europa- bzw. weltweite Transformation von der Industrie- zur Dienstleistungsgesellschaft, die den industriellen Strukturwandel in neuen Formen fortsetzt und bei der auf Grund von Rationalisierungen in Landwirtschaft und Industrie, auf Grund gesellschaftlicher Veränderungen (Entstehung eines Freizeitsektors, Umwandlung von innerfamiliären Erziehungs-, Sozial- und Pflegetätigkeiten in Lohnarbeit), auf Grund höherer Anforderungen in der Arbeitswelt (Bildungs- und Ausbildungswesen) sowie auf Grund steigenden Regelungs- und Organisationsbedarfs (staatliche und private Verwaltung) die Bedeutung der Dienstleistungen überproportional wächst. Charakteristisch für die Dienstleistungsgesellschaft ist die Herausbildung überkomplexer Strukturen bei gleichzeitiger Eindimensionalität: Die heutigen Wirtschaftsbetriebe operieren im Rahmen höchster Spezialisierungen weltweit in einem kleinen Marktsegment, wobei sie ihre Partner und Konkurrenten in anderen Erdteilen besser kennen als ihre Nachbarn in der eigenen Gemeinde. Eine analoge Entwicklung läuft im gesellschaftlichen Bereich $a b$, wo in den Zentren der Dienstleistungsgesellschaft die Familie und die gemeinsamen verbindlichen Werte zerfallen und sich die Gesellschaft in einzelne Interessen-, Berufs- und Altersklassen auflöst. Wurde die Agrargesellschaft auf positive Weise durch gemeinsame (Land-)Wirtschaftsinteressen und die Industriegesellschaft auf negative Weise durch den Gegensatz zwischen Kapital und Arbeit zusammengehalten, so zerfällt die Dienstleistungsgesellschaft in einzelne Segmente, die konzeptionell immer unverbundener und unverbindlicher nebeneinanderstehen, obwohl sie faktisch stark (negativ) interagieren. Die Bezeichnung «Postmoderne» für einen Wertepluralismus, der keine gesellschaftlichen Verbindlichkeiten mehr kennt, kennzeichnet diese Situation gut aus philosophischer Sicht.

Auf der räumlichen, also geographischen Ebene ist die Dienstleistungsgesellschaft europa- bzw. weltweit dadurch gekennzeichnet, daß die funktionalen Spezialisierungen mit räumlichen Segregationen und Entmischungsprozessen verbunden sind: Die Bereiche Wohnen, Arbeit und Freizeit werden funktional und räumlich getrennt und entwickeln jeweils eine eigenständige Dynamik. Dabei steigt der Flächenverbrauch stark an, weil das private Konsumniveau hoch geworden ist (steigende Wohn- und Grundfläche pro Kopf), weil die modernen Industrie- und Dienstleistungsbetriebe einen großen Platzbedarf besitzen, weil durch die räumlichen Trennungen ein starker Verkehr erzeugt wird, der einen großen Flächenbedarf besitzt, und weil räumlich getrennte Nutzungsarten keine komplementären Flächennutzungen mehr erlauben, so daß zu beliebigen Tages-, Wochen- oder Jahreszeiten jeweils bestimmte Teilflächen leerstehen. Die Folge ist ein übergroßer Flächen- und Landschaftsverbrauch mit extremen Flächennutzungskonkurrenzen um den immer knapperen «Boden».

\section{Die räumliche Entwicklung der Dienstleistungsgesellschaft}

Dadurch wird der Stadt-Land-Gegensatz, das räumliche Entwicklungsmuster der Industriegesellschaft, überall aufgehoben: Auch wenn durch die industrielle Revolution das Land zur Provinz degradiert wurde und sich aller «Fortschritt» in der Stadt konzentrierte, so blieben doch zwei unterschiedliche Lebensmuster erhalten, die beide Male durch enge räumliche Verflechtungen und Identitäten zwischen Wohn-, Arbeits- und Freizeitwelt charakterisiert waren und die sich in unverwechselbaren Kulturlandschaften (städtischer und ländlicher Art) ausdrückten. Im Verlauf der Tertiarisierung wird die Innenstadt monostrukturell zum Dienstleistungszentrum und der randstädtische Bereich zum Gewerbe- und Industriestandort umgewandelt, wobei die verbleibenden Stadtbezirke durch den täglichen Pendlerverkehr erstickt und entwertet werden. Die Wohnnutzung wird aufs Land verdrängt, wo die Dörfer ihren «dörflichen», d. h. bäuerlich-multifunktionalen Charakter verlieren und bloß noch Schlafstandort einer Pendlerbevölkerung werden. Am Ende steht eine anonyme Suburbanisationslandschaft, die weder städtischen noch ländlichen Charakter besitzt und die auf Grund ihrer kalten Funktionalität nicht mehr als «Heimat» erlebt werden kann.

Jenseits der Suburbanisationsräume setzen sich auf dem Land neue Funktionsdifferenzierungen und -entmischungen durch: Gebiete mit guter naturräumlicher Eignung und guter Agrarstruktur bilden Agrarmonostrukturen mit großflächigen Agrarsteppen und Agroindustriebetrieben aus. Gebiete mit abwechslungsreicher Landschaft, guter Verkehrserschliessung und Infrastruktur entwickeln sich zu Tourismusregionen mit Monostruktur, und der gesamte «Rest» degradiert zu strukturschwachen Regionen, in denen jetzt auch die Landwirtschaft, die letzte Wirtschaftsaktivität, zusammenbricht. Im dicht genutzten Europa bilden solche strukturschwachen Regionen aber ein räumliches Vakuum, das all jene Nutzungen anzieht, für die in den Suburbanisationslandschaften kein Platz mehr ist: Militärische Übungsplätze, große Stauseen, Müll- und Sondermülldeponien oder die Ausweisung von Naturschutzgebieten als «ökologische Ausgleichsräume».

Am Ende sind die unverwechselbaren Kulturlandschaften zerstört, und es stehen hochspezialisierte und monostrukturell geprägte Landschaften neben- 
einander, die überall in Europa identisch aussehen und die den Menschen immer weniger "Heimat» sein können.

\section{Die Gefahr der Tertiarisierung für die Schweiz}

Die Tertiarisierung ist in der Schweiz dadurch charakterisiert, daß keine äußerlich sichtbaren «Brüche» (Kriegszerstörungen, Flüchtlingsströme) erkennbar sind und die Transformation von Wirtschaft und Gesellschaft nur sehr allmählich geschieht. Besonders bemerkenswert ist dabei, daß das am Ende der 30er Jahre entwickelte kulturelle Selbstbewußtsein, das einer geistigen Abwehr verpflichtet ist («Landi '39»), sowohl die Nachkriegszeit als auch das Jahr 1968 überdauert und erst am Ende der 80er Jahre ins Wanken gerät (Armee-Abstimmung, Fichen-Affäre, Fall Kopp u. a.), so dass ein die Tertiarisierung widerspiegelndes «postmodernes» $\mathrm{Be}$ wußtsein noch aussteht. Trotzdem haben sich erhebliche Veränderungen durchgesetzt: Das Dienstleistungszentrum Zürich ist im Verlauf der Nachkriegszeit so groß geworden, daß es die Rolle eines die ganze Schweiz dominierenden Zentrums einzunehmen beginnt, wodurch der föderative Charakter der Eidgenossenschaft in Frage gestellt wird.

Angesichts der Tatsache, daß die Schweiz genau auf der stark expandierenden europäischen Wirtschaftsachse London-Rhein-Oberitalien liegt und $\mathrm{da} B$ die Wirtschaftskräfte, die heute von außen in die Schweiz hineinwirken, nicht mehr nationale Firmen, sondern multinationale Konzerne sind, wird es der kleinen, zentral gelegenen und gut erreichbaren Schweiz sehr schwerfallen, sich diesem Einfluß zu entziehen. Die Entwicklung tendiert daher dahin, aus dem Mittelland eine durchgehende Suburbanisationslandschaft (nebst einigen Agrarsteppen und ökologischen Ausgleichsräumen) und aus den Gebirgsräumen monostrukturelle Freizeiträume (nebst einigen Industrie- und Dienstleistungszentren) zu machen.

Die zahlreichen ländlichen und städtischen Kulturlandschaften, die heute noch in der Schweiz wesentlich besser erhalten sind als in den übrigen europäischen Staaten, dürften dadurch in absehbarer Zeit zerstört werden. Damit wird auch die je besondere Beziehung des Menschen zu sich selbst, zu seinen Mitmenschen, zu seiner Geschichte und Umwelt zerstört, und die auf die reine Funktionalität reduzierten Menschen werden im räumlichen und kulturellen Sinne boden- und heimatlos. Damit zerfiele ein zentrales Charakteristikum der Schweiz, nämlich das gleichberechtigte Neben- und Miteinander verschiedener historisch gewachsener Wirtschaftsund Lebensmodelle in einem Staat, und die Schweiz entwickelte sich zu einer gesichts- und identitätslosen europäischen Region.
Ähnlich wie in den Jahren 1848, 1874 oder 1939 steht die Schweiz heute wieder vor einer Herausforderung, die nur mit einem Paradigmenwechsel, einem neuen Selbstverständnis gelöst werden kann. ${ }^{1}$ Dabei geht es nicht um einen Bruch mit den Werten der Vergangenheit, sondern um ihre Neubewertung, um auf die aktuellen Probleme eine helvetische Antwort zu finden.

Dabei kann sich die Schweiz auf verschiedene europaweite Gegenbewegungen (Wiederaufwertung der ländlichen Räume, Revitalisierung der Innenstädte) sowie auf wichtige nationale Ansätze stützen, die fundierte Gegenpositionen formuliert haben wie in der Landwirtschaftspolitik (das Bewußtsein der multifunktionalen Aufgabe der Landwirtschaft ist hier größer als sonst in Europa), in der Regionalpolitik (die Probleme der räumlichen Monostrukturen wurde hier sehr früh erkannt), in der Verkehrspolitik (bewußtes Gegensteuern gegen die EGVerkehrspolitik) und in der Umweltpolitik. Diese müßten dann mit den spezifisch schweizerischen Strukturen (föderalistischer Staatsaufbau, Mehrsprachigkeit und kulturelle Vielfalt, Konsensdemokratie) auf neue Weise verbunden werden. Leitidee dabei müßte es sein, die europäische Tertiarisierung in der Schweiz so zu gestalten, daß die neuen wirtschaftlichen Möglichkeiten, die sich daraus ergeben, bewußt mit den traditionellen Wirtschaftsbereichen (v. a. Landwirtschaft und Handwerk) vernetzt werden, so daß lebensfähige multifunktionale Lebensräume bzw. Kulturlandschaften erhalten bzw. neu geschaffen werden, die für ihre Bewohner Heimat darstellen und die ihre jeweilige Umwelt sorgsam nutzen, ohne sie zu zerstören.

Angesichts der europa- bzw. weltweiten Dynamik der Tertiarisierung wäre die Schweiz überfordert, allein auf ihrem Territorium multifunktionale Kulturlandschaften zu erhalten. Dies ist allerdings kein ausschließlich schweizerisches Problem, sondern betrifft ganz Europa. Daher müssen europaweit neue wirtschaftliche und politische Rahmenbedingungen geschaffen werden. Die Schweiz kann heute nur dann ihre schweizerische Identität bewahren, wenn sie sich auf die europäische Ebene begibt und für ein «Europa der Regionen» engagiert: Nur im regionalen Rahmen können Wirtschaft, Kultur und Umwelt multifunktional so miteinander verbunden werden, daß lebensfähige und lebenswerte Kulturlandschaften entstehen, die als Synthese zwischen Mensch und Natur und zwischen Vergangenheit und Gegenwart Zukunft ermöglichen.

\section{Anmerkung \\ 1 RHINOW, R. (1991): Braucht die Schweiz Visionen? In: Neue Zürcher Zeitung, Nr. 9 vom 12./13.1.1991, S. 21.}

